\title{
Capecitabine-induced cardiotoxicity: more evidence or clinical approaches to protect the patients' heart?
}

\author{
Caterina Fontanella' \\ Marianna Aita' \\ Marika Cinausero' \\ Giuseppe Aprile' \\ Maria Grazia Baldin² \\ Veronica Dusi ${ }^{3}$ \\ Chiara Lestuzzi ${ }^{4}$ \\ Gianpiero Fasola' \\ Fabio Puglisi ${ }^{1,5}$ \\ 'Department of Oncology, University \\ Hospital of Udine, Udine, Italy; \\ ${ }^{2}$ Department of Cardiology, \\ Palmanova General Hospital, \\ Palmanova, Italy; ${ }^{3}$ Department of \\ Cardiology, Fondazione IRCCS \\ Policlinico San Matteo, Pavia, Italy; \\ ${ }^{4}$ Department of Cardiology, Centro \\ di Riferimento Oncologico, National \\ Cancer Institute, Aviano, Italy; \\ ${ }^{5}$ Department of Medical and Biological \\ Sciences, University of Udine, \\ Udine, Italy
}

This article was published in the following Dove Press journal:

OncoTargets and Therapy

29 September 2014

Number of times this article has been viewed

\begin{abstract}
Fluoropyrimidines, such as capecitabine and 5-fluorouracil, may cause cardiac toxicity. In recent years, the incidence of this side effect has increased and it is expected to further rise due to the population aging and the disproportionate incidence of breast and gastrointestinal cancers in older individuals. The spectrum of cardiac manifestations includes different signs and symptoms and the diagnosis may be difficult. Here, we report the case of a 43 -year-old woman with advanced breast cancer who was rechallenged with a capecitabine-based regimen after experiencing a cardiac adverse event during the first fluoropyrimidine exposure. This real-practice case serves as a springboard for discussion about the current evidence on differential diagnosis of capecitabine-related cardiac toxicity, its risk factors, and the underpinning mechanisms of early onset. Moreover, we discussed whether a rechallenge with fluoropyrimidines could be safe in patients who had experienced a previous cardiac adverse event.
\end{abstract}

Keywords: risk factors, clinical manifestation, rechallenge

\section{Introduction}

Capecitabine, an oral prodrug of 5-fluorouracil (5-FU), is an orally available fluoropyrimidine analog selectively activated through a three-step enzymatic process. The final transformation is catalyzed by thymidine phosphorylase, an enzyme with higher activity in tumors than in normal tissues. ${ }^{1}$ Thus, after its administration, 5-FU levels are higher in tumor cells than in the systemic circulation. ${ }^{1,2}$

Capecitabine has a well-documented safety profile; the most commonly reported side effects are hand-foot syndrome (17\%), diarrhea (17\%), and nausea (15\%), but all these toxicities are usually manageable and reversible. ${ }^{1,3}$ Although well tolerated, both capecitabine and its parental compound may induce cardiotoxicity, whose incidence is increasing due to the aging of the population. ${ }^{4}$ The spectrum of cardiac effects includes blood pressure disorders, acute coronary syndromes, arrhythmias, heart failure with cardiogenic shock, and even sudden death. ${ }^{5}$

Our real-practice paradigmatic case of capecitabine-related cardiotoxicity is helpful in summarizing the current evidence on this important safety concern (differential diagnosis, mechanisms of onset, risk factors) and serves as an example to discuss the safety of fluoropyrimidine rechallenge after a first cardiac episode.

\section{Presentation of case}

Correspondence: Caterina Fontanella University Hospital of Udine, Department of Medical Oncology, P le Santa Maria della Misericordia 15, 33100 Udine, Italy Email fontanella.caterina@spes.uniud.it

A 43-year-old premenopausal woman was diagnosed in July 2006 with a locally advanced (stage IIIA, cT3 cN1 M0), ${ }^{6}$ hormone receptors-positive (estrogen receptor 
[ER] $40 \%$ of positive cells, progesterone receptor [PgR] $50 \%$ of positive cells), and human epidermal growth factor receptor (HER)2-positive (immunohistochemistry score 3+) invasive ductal carcinoma of the left breast. The Ki67/MIB-1 immunostaining was positive in $32 \%$ of cancer cells. At the time of primary diagnosis, the patient was a light smoker (less than five packs per year) with a family history of hypertension and ischemic heart disease; no other risk factors for cardiac disease were reported.

On August 2, 2006, the patient was randomized into the neoadjuvant ECTO II (European Cooperative Trial in Operable breast cancer-second) $\operatorname{trial}^{7}$ and she was allocated to receive exemestane $25 \mathrm{mg}$ /day orally plus four cycles of doxorubicin $60 \mathrm{mg} / \mathrm{m}^{2}$ intravenously (iv); cyclophosphamide $600 \mathrm{mg} / \mathrm{m}^{2}$ iv at 3-week intervals (AC) followed by four cycles of paclitaxel $100 \mathrm{mg} / \mathrm{m}^{2}$ iv on days 1 and 8 ; and capecitabine $1,850 \mathrm{mg} / \mathrm{m}^{2}$ orally in divided doses from day 1 to 14 at 3-week intervals (TX).

The AC regimen was well tolerated, without any cardiovascular complaint.

In November 2006, during the first cycle of TX regimen, she presented at the Emergency Ward of the University Hospital of Udine, Italy, to investigate a recurrent, sharp, nonradiating retrosternal chest pain, which had started 24 hours previously. The pain lasted up to 20 minutes and was unrelated to meals and exercises, whereas it was relieved by a change of position. Blood pressure, pulse rate, and body temperature were normal. No pericardial friction rub, added heart sounds, or other abnormalities were observable through auscultation. Blood count, lactate dehydrogenase, transaminases, serum C-reactive protein (CRP), serum creatine phosphokinase, and creatine phosphokinase-MB fraction were all within normal limits. However, the plasma troponin I (TnI) concentration was $0.27 \mathrm{ng} / \mathrm{mL}$ (normal levels $\leq 0.04 \mathrm{ng} / \mathrm{mL}$ ) and the continuous 12-lead electrocardiogram (ECG) Holter monitoring showed concave ST segment elevation in multiple leads, biphasic $\mathrm{T}$ wave inversion, and peaked T wave elevation (Figure 1). The ratio of ST segment elevation to $\mathrm{T}$ wave amplitude in lead V6 was 0.4. The electrocardiographic findings suggested the possibility of an acute pericarditis, although elevated $\mathrm{TnI}$ concentration was not concordant with the diagnosis.

A second cardiac evaluation was performed with a two-dimensional echocardiogram (2D Echo) that showed inferoposterior hypokinesis, with preserved global ventricular size and function; mild aortic and mitral regurgitation were also observed, with normal left atrium and absence of pericardial effusion. The final diagnosis was acute myopericarditis, and anti-inflammatory treatment with acetylsalicylic acid $800 \mathrm{mg}$ twice a day for 1 month was started. The following day, white blood-cell count and serum CRP were confirmed to be normal, and plasma TnI level was $0.12 \mathrm{ng} / \mathrm{mL}$. Two days later, a multigated acquisition scan showed normal regional kinetics, with a left ventricular ejection fraction of $67 \%$, and normal ECG tracing. However, the TX neoadjuvant treatment was interrupted and the patient was referred for breast surgery.

The patient improved soon after capecitabine discontinuation. In light of this prompt resolution, a fluoropyrimidine-induced cardiotoxicity was strongly suspected. The investigators did not perform a myocardial biopsy due to the invasive nature of this procedure.

After radical left mastectomy, a ypT2 ypN1mi, hormone receptors-positive (in this case, a semiquantitative evaluation of ER and PgR was calculated using the H-score: ER H-score 30; PgR H-score 15), HER2-positive tumor was detected, with a Ki67/MIB-1 proliferation index that resulted positive in 32\% of cancer cells. Both ECG and 2D Echo showed no abnormalities, with preserved left ventricular size and function, and a left ventricular ejection fraction of $64 \%$. Therefore, the patient was offered adjuvant endocrine treatment and trastuzumab $6 \mathrm{mg} / \mathrm{kg}$ ( $8 \mathrm{mg} / \mathrm{kg}$ loading dose) iv every 3 weeks for 1 year. For safety reasons, trastuzumab was always infused within 90 minutes. From February 2007 to February 2008, the patient received a total of 18 trastuzumab administrations, without any cardiac complications.

Also, the patient underwent irradiation of the lymphatic drainage area, while excluding chest wall irradiation.

\section{Second cardiac event}

One year later, the patient was diagnosed with sternum and liver metastases and received first-line treatment with six cycles of docetaxel plus trastuzumab, followed by trastuzumab single agent. At disease progression, a secondline chemotherapy with lapatinib and capecitabine was planned.

Fluoropyrimidines rechallenge was attempted with reduced dose of capecitabine: $1,000 \mathrm{mg} / \mathrm{m}^{2} /$ day, instead of $2,000 \mathrm{mg} / \mathrm{m}^{2} /$ day, ${ }^{8}$ orally from day 1 to day 14 every 3 weeks. Moreover, a cardiac surveillance with daily 12-lead ECG, 24-hour ECG Holter monitoring on the 4th day of chemotherapy, and treadmill stress test on the 5th day was planned in order to detect early signs of cardiac toxicity.

On March 1, 2010, the patient received the first dose of lapatinib $1,250 \mathrm{mg}$ /day continuously plus capecitabine $1,000 \mathrm{mg} / \mathrm{m}^{2} /$ day, from day 1 to day 14 , every 21 days. 


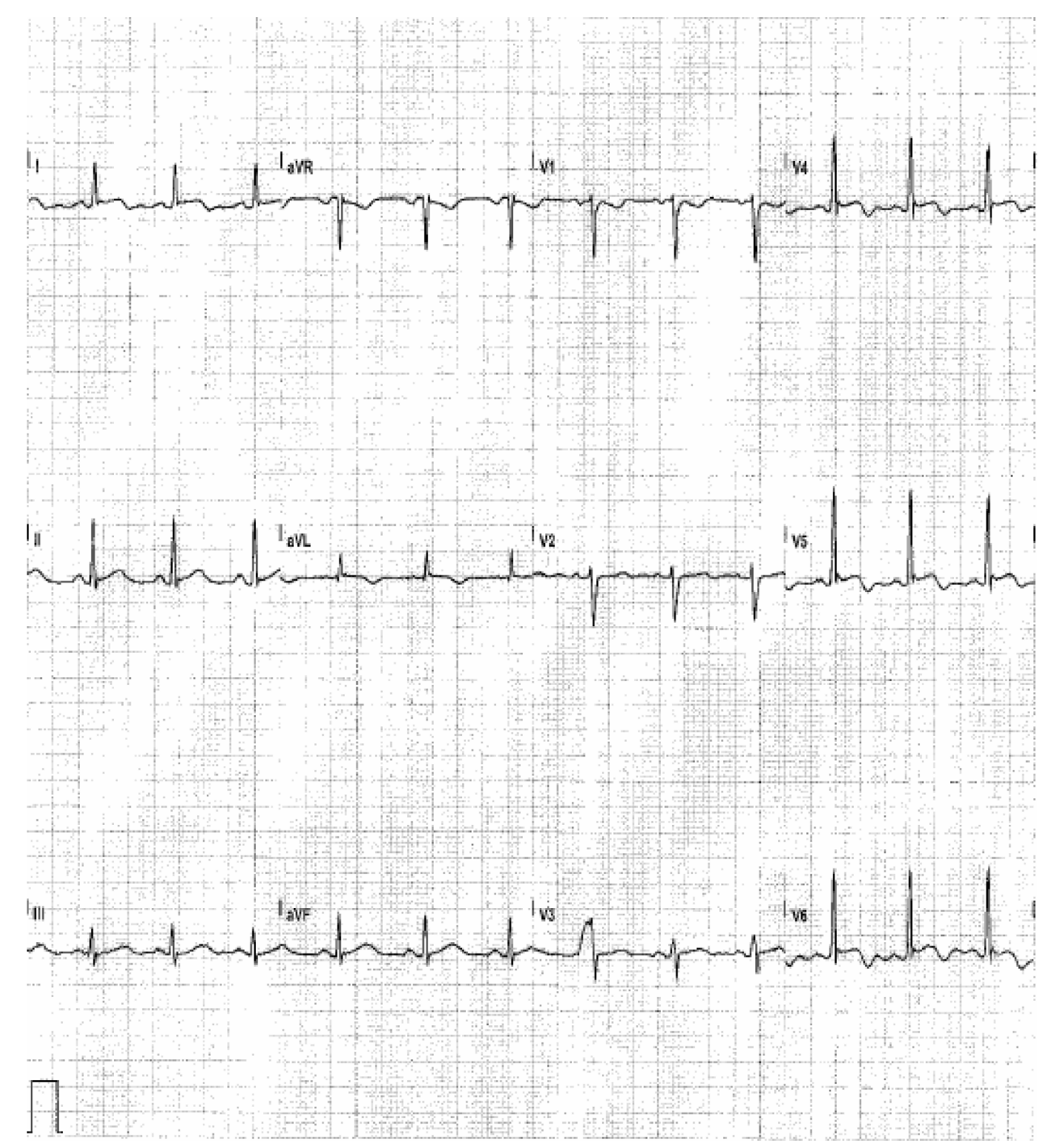

Figure I First cardiac event.

Notes: Traces from the I2-lead ECG $(25 \mathrm{~mm} / \mathrm{s}, 10 \mathrm{~mm} / \mathrm{mV})$ showed transient abnormalities. Peaked T wave and ST segment elevation were associated with thoracoabdominal discomfort. I, II, and III = limb leads; VI to V6 = precordial leads.

Abbreviations: aVF, lead augmented vector foot; aVL, lead augmented vector left; aVR, lead augmented vector right; ECG, electrocardiogram.

Five days later, during 24-hour ECG Holter monitoring, she was admitted at the Emergency Ward of a General Hospital (20 miles away from our University Hospital) due to short recurrent episodes of atypical chest and abdominal pain, associated with jugular constriction. No abnormalities were underlined with physical examination, 12-lead ECG, and 2D Echo. The plasma TnI concentration was less than $0.005 \mathrm{ng} / \mathrm{mL}$, within the range of normality.

The Holter monitoring showed electrocardiography features consistent with Prinzmetal's angina: heart rate ranged from 45 to 137 beats/minute and transitory ischemic ECG changes, such as ST segment depression and elevation, and $\mathrm{T}$ wave negative inversion or peaked elevation (Figure 2A-B). Particularly, an infero-antero-lateral $4 \mathrm{~mm}$ ST segment elevation was observed from $7.20 \mathrm{am}$ to $7.50 \mathrm{am}$, while the patient was asymptomatic (Figure 3 ). The patient received amlodipine $10 \mathrm{mg}$, sublingual nitrates, and acetylsalicylic acid $100 \mathrm{mg}$ and was discharged 24 hours later.

In order to rule out atherosclerotic coronary artery disease and to better define the first episode of cardiotoxicity, the patient underwent coronary artery computed tomography, which showed normal arteries, and cardiac magnetic resonance, which did not show any sign of acute or previous myocarditis.

According to the strong suspicion of capecitabine-induced cardiotoxicity, the second-line treatment was changed to vinorelbine $25 \mathrm{mg} / \mathrm{m}^{2}$ plus trastuzumab $2 \mathrm{mg} / \mathrm{kg}$ weekly.

\section{Discussion}

Differential diagnosis between capecitabine-related cardiotoxicity and other cardiac abnormalities. 

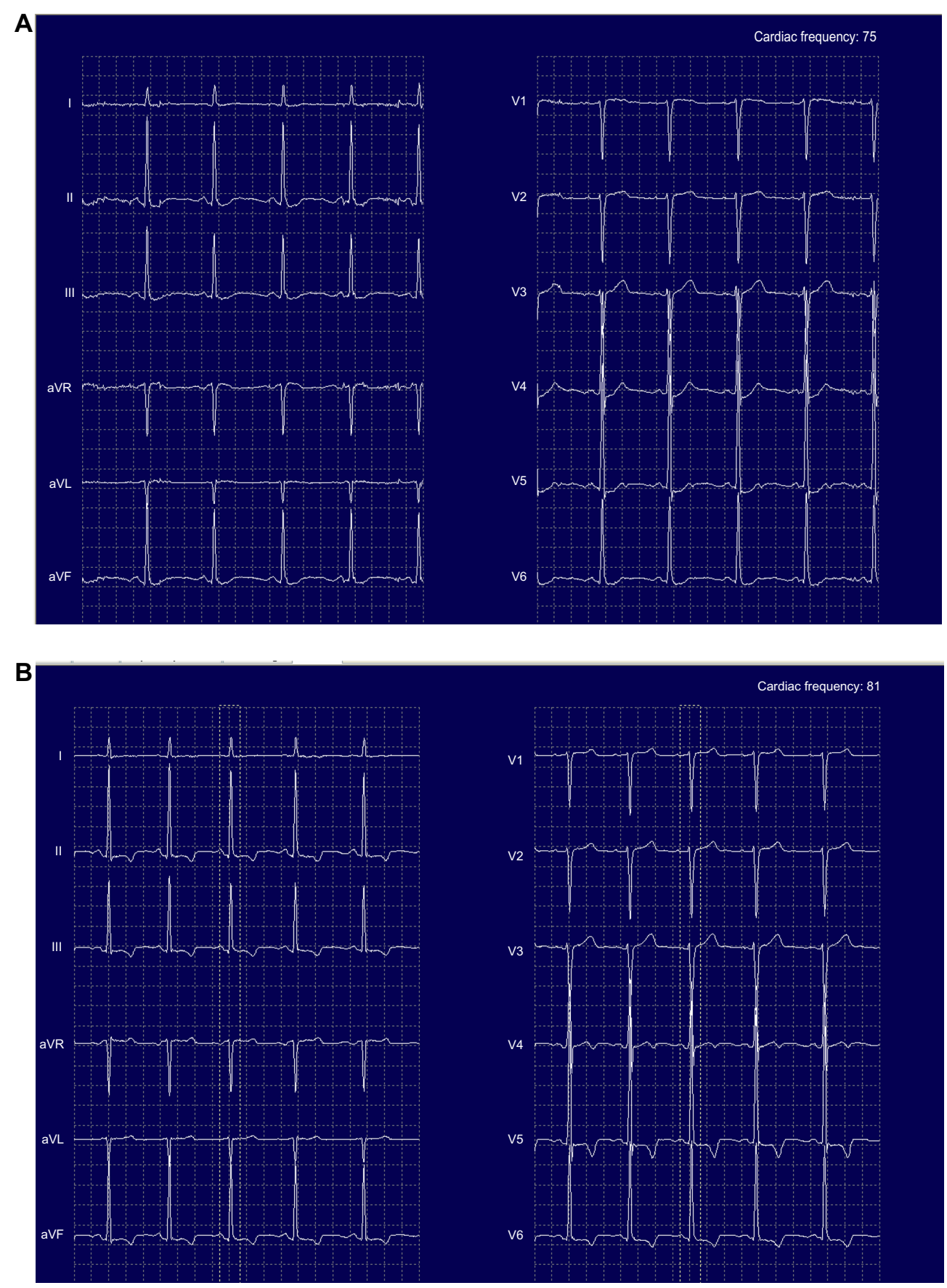

Figure 2 Second cardiac event.

Notes: Traces from the continuous 12-lead 24 hours ECG Holter monitoring $(25 \mathrm{~mm} / \mathrm{s}, 10 \mathrm{~mm} / \mathrm{mV})$ showed transient abnormalities. (A) Heart rate 75 bpm: ST segment depression; (B) Heart rate 8I bpm: Asymptomatic T wave negative inversion. I, II, and III = limb leads; VI to V6= precordial leads.

Abbreviations: aVF, lead augmented vector foot; aVL, lead augmented vector left; aVR, lead augmented vector right; ECG, electrocardiogram.

\section{First cardiac event}

During neoadjuvant treatment, clinical symptoms, TnI elevation, ECG findings (widespread ST segment elevation and T wave inversion without development of Q waves), and regional hypokinesia at $2 \mathrm{D}$ Echo suggested a possible diagnosis of acute myopericarditis. ${ }^{9}$ Nevertheless, blood tests showing a normal leukocyte and eosinophil count, with no elevation of CRP, were against this hypothesis and supportive of capecitabine-induced cardiotoxicity. Even if the hypothesis of acute myopericarditis would be accepted, the viral etiology could not be excluded, since serum antibodies against the most common viruses (ie, echovirus and coxsakievirus) were not checked.

Signs and symptoms experienced by the patient may also suggest an acute coronary syndrome or a Takotsubo syndrome, 


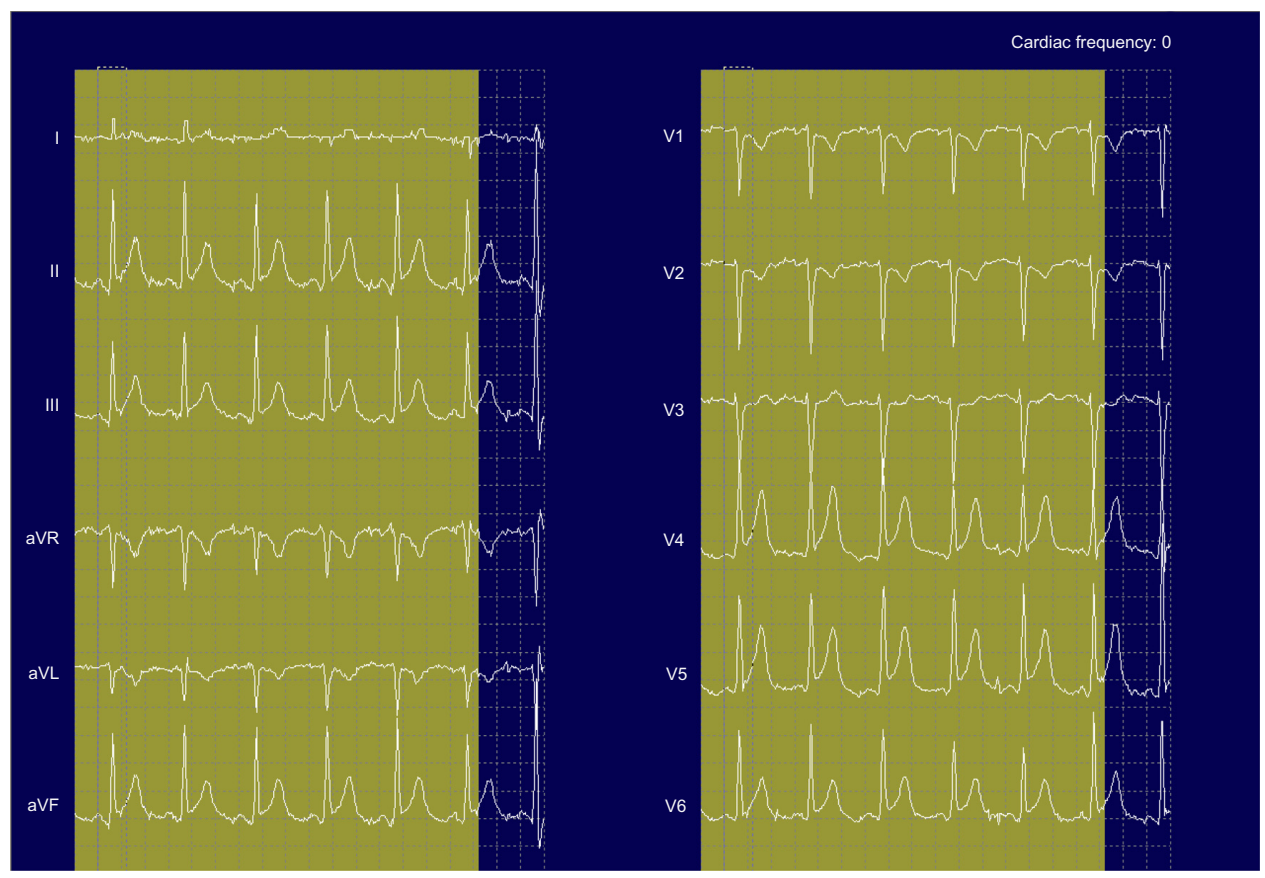

Figure 3 Second cardiac event.

Notes: Trace from the continuous I2-lead 24 hours ECG Holter monitoring ( $25 \mathrm{~mm} / \mathrm{s}, 10 \mathrm{~mm} / \mathrm{mV})$ showed: Heart rate $100 \mathrm{bpm}$, $4 \mathrm{~mm}$ asymptomatic ST segment elevation. I, II, and III = limb leads; VI to V6 = precordial leads.

Abbreviations: aVF, lead augmented vector foot; aVL, lead augmented vector left; aVR, lead augmented vector right; ECG, electrocardiogram.

both linked with 5-FU and capecitabine administration. ${ }^{10-14}$ In particular, capecitabine has been reported to trigger apical and midventricular Takotsubo syndrome characterized by ECG signs of ST elevation myocardial infarction, severe left ventricular dysfunction, and cardiogenic shock. ${ }^{13}$ Unlike acute myocardial infarction, Takotsubo syndrome is generally reversible in a short period and it is not associated with significant coronary artery disease. Catecholamine-induced cardiotoxicity, microvascular dysfunction, and a perfusion/ metabolism mismatch, such as in postischemic myocardial stunning, are the most common pathogenic events involved in Takotsubo syndrome. ${ }^{14}$ Of note, acute 2D Echo pattern showed inferoposterior hypokinesis and it was incongruous with apical and midventricular transient left ventricular dysfunction syndrome.

Delayed anthracyclines toxicity could have been also considered, since anthracyclines have been occasionally associated with the occurrence of a myocarditis-pericarditis syndrome. ${ }^{15}$ However, this rare syndrome has been described within 1 month from the first anthracyclines administration. ${ }^{16}$ In our patient, the cardiac symptomatology appeared more than 3 months after the first infusion of doxorubicin and had a benignant course; moreover, both the ECG tracing and the echocardiography were normal at the end of anthracyclinebased chemotherapy.

\section{Second cardiac event}

The second episode was characterized by atypical chest pain without detectable changes on ECG tracing. However, 24-hour Holter monitoring documented a number of transitory ischemic ST segment deviation and $\mathrm{T}$ wave abnormalities.

Transitory ST segment elevation or depression and $\mathrm{T}$ wave inversion, especially if they occurred in the early morning hours, should be considered consistent with vasospastic angina. ${ }^{17,18}$ As demonstrated in animal models and in human studies, ${ }^{19,20}$ arterial vasospasm is one of the possible mechanisms of 5-FU related angina. During a capecitabinerelated coronary spasm, patients may experience angina-like chest pain ${ }^{21,22}$ and ventricular fibrillation. ${ }^{23,24}$ Moreover, the diagnosis of vasospastic angina could be consistent with the hypothesis of an acute coronary syndrome also in the first cardiac event presented during neoadjuvant treatment.

Nevertheless, similar ST segment abnormalities may also be observed in acute myocardial infarction, acute pericarditis, myopericarditis, and Prinzmetal's angina. ${ }^{25}$

The milder symptoms during the second episode are justified by the shorter exposure time and the lower dose of capecitabine administrated. In fact, the incidence of cardiac toxicity of fluoropyrimidines depends on both the cumulative dose of drug stored within the heart and 
the clearance of the active metabolites from tissues in the individual patient. For this reason, the cardiotoxic effects may even last days after withdrawing the drug. ${ }^{26}$ The diffuse hypokinesia detected with 2D Echo and the elevated TnI plasma concentration that occurred only in the first cardiac event may be justified by the long duration of the first vasospastic event.

\section{Incidence and molecular mechanisms of cardiotoxicity}

The chance for cardiac toxicity during fluoropyrimidinesbased treatment ranges from $1.2 \%$ to $18 \%$ with a mortality rate between $2.2 \%$ and $13.3 \%,{ }^{27}$ although larger trials have suggested an incidence of symptomatic cardiotoxicity of $1.2 \%-4.3 \%$ during treatment. A large meta-analysis on breast and colorectal cancer patients treated with fluoropyrimidines ${ }^{28}$ confirmed this observation, revealing a similar low incidence $(3 \%)$ of symptomatic 5 -FU- and capecitabine-related events.

Interestingly, in a prospective study testing the potential development of cardiac-related symptoms during fluoropyrimidine-based chemotherapy, symptomatic ECG abnormalities were significantly more frequent in patients treated with 5-FU continuous 24-hour infusion and with capecitabine than in those receiving short infusion of 5-FU. On the other hand, no difference was observed between the incidence of capecitabine and 5-FU 24-hour infusion cardiotoxicity. ${ }^{29}$

The finding of a shared, schedule-associated toxic effect on myocardium is consistent with capecitabine pharmacodynamics, with a prolonged exposure of tumor cells to the drug mimicking continuous intravenous 5-FU infusion. ${ }^{30}$ Even if 5-FU plasma half-life is very short, active metabolites are retained at tissue level, both in tumor and in the heart, as demonstrated by pharmacokinetics studies. ${ }^{31}$

The mechanism of cardiotoxicity of fluoropyrimidines remains poorly defined. One of the documented causes of cardiotoxicity is the effect on vascular endothelium that involves endothelial nitric oxide (NO) synthase; 5-FU appeared to induce direct vasoconstriction on smooth muscle cells, which was shown to be protein kinase C-dependent. ${ }^{32}$ A reduced synthesis of NO leads to coronary spasms and endothelium-independent vasoconstriction via the protein kinase pathway. ${ }^{33}$ However, it has been clarified more than 20 years ago that neither calcium channel blockers $^{34}$ nor nitrates ${ }^{35}$ can reduce the risk of fluoropyrimidines-related cardiotoxicity; thus, other pathogenic mechanisms need to be involved apart from the reduced concentration of NO.
Animal models provided the evidence of an increased endothelial thrombogenicity during 5-FU treatment, indicating a toxic effect of 5-FU on endothelial cells. ${ }^{36}$ Moreover, given the global systolic dysfunction related to fluoropyrimidines cardiotoxicity, the possibility of a direct toxic action on the whole myocardium rather than focused coronary artery damages has also been evaluated. ${ }^{37}$ Recently, a preclinical model showed an apoptotic effect induced by the increase of intracellular reactive oxygen species in cardiomyocytes exposed to 5-FU. This observation suggested that 5-FU induces cardiomyocytes damage by oxidative stress. ${ }^{27}$

Synergic cardiotoxicity effect between capecitabine and other agents concomitantly administered has also been speculated. A retrospective study showed that cardiotoxicity was more frequently reported in patients receiving capecitabine plus taxanes than in those treated with capecitabine single agent (3.7\% versus $1.9 \%$ ), although the difference did not reach statistical significance. ${ }^{38}$ In our patient, cardiac toxicity was evident both in the TX schedule and in association with lapatinib.

\section{Risk factors for cardiotoxicity}

Having a history of ischemic heart disease is the strongest predisposing factor for fluoropyrimidines-induced cardiotoxicity. ${ }^{5}$ A prospective cohort study conducted in 35 hospitals found that patients with preexisting cardiovascular disease have a significantly increased risk for cardiotoxicity (risk ratio $[R R]=6.83$ ) compared to those without baseline cardiac disease. ${ }^{39}$ Moreover, patients receiving concomitant calcium channel blockers $(\mathrm{RR}=4.75)$ and those receiving nitrates $(\mathrm{RR}=9.18)$ have an increased risk for cardiotoxicity. ${ }^{39}$ The importance of preexisting cardiac disorders as a risk factor for cardiotoxicity was also highlighted in a retrospective studies specifically focused on capecitabine. ${ }^{38,40}$ The increased expression of thymidine phosphorylase, the key enzyme involved in the conversion of capecitabine to 5-FU, in both atherosclerotic plaques and tumor tissues ${ }^{41}$ may justify the higher prevalence of capecitabine-induced cardiotoxicity in patients with ischemic heart disorders.

Recently, a study focused on patients treated with 5-FU continuous 24-hour infusion showed that the complaint of chest or jaw pain and dyspnea in daily life during the first day of infusion is highly predictive of abnormalities during treadmill stress test. ${ }^{42}$

A link between cardiotoxicity and previous chest radiotherapy or baseline renal function impairment has also been suggested. ${ }^{38}$ Conversely, no association with family history, hypertension, and smoking was found. ${ }^{29,38,43}$ 
At the time of first diagnosis, our patient was a fully healthy young woman with no personal or familiar history of cardiac disease. After mastectomy she underwent irradiation of the lymphatic drainage area, while excluding chest wall irradiation. Moreover, during metastatic treatment, the absence of atherosclerotic coronary artery disease was documented with coronary artery computed tomography and cardiac magnetic resonance. In accordance with literature data, she had no established risk factors for fluoropyrimidinesrelated cardiac toxicity; thus, there were no consistent reasons to avoid capecitabine treatment.

\section{Clinical manifestation of cardiotoxicity}

In a retrospective analysis of 688 patients consecutively treated for colorectal or gastric cancer, all cases of capecitabine cardiotoxicity occurred during the first cycle, with a median time for first occurrence of only 4 days from the start of treatment (range from 2 to 15 days). ${ }^{38}$

In 2011, Koca et al ${ }^{40}$ reported a 34.6\% level of incidence for symptomatic cardiac events among 52 cancer patients treated with capecitabine either as single agent or in combination with other drugs; palpitation (23.1\%) and chest pain $(9.6 \%)$ were the most common clinical manifestations. However, the percentage of patients who experienced symptomatic toxicity significantly decreased in trials that involved a larger number of patients. ${ }^{29,38,43}$ Taken together, these trials showed an overall incidence of symptomatic cardiotoxicity from $4.0 \%$ to $6.5 \%$ and identified chest pain as the most frequent reported symptom. Sudden cardiac death during fluoropyrimidines treatment is a very rare event, accounting for less than $1 \%$ of cardiotoxicity clinical manifestation. ${ }^{29,43}$

Electrocardiographic abnormalities were documented by 12-lead ECG tracing in one study involving patients treated with capecitabine. ${ }^{40}$ One-third of patients $(32.6 \%)$ were found to have new-onset ECG alterations: $25.0 \% \mathrm{ST}$ segment deviation, $21.1 \%$ sinus tachycardia, and $19.2 \%$ prolonged QTc interval.

No study was conducted to assess the level of plasma troponins during capecitabine treatment. However, three studies ${ }^{44-46}$ were conducted in patients treated with 5-FU with discordant results; only Holubec et a ${ }^{16}$ reported a pathologic plasma TnI value $(>0.04 \mathrm{ng} / \mathrm{mL})$ in $57 \%$ of all enrolled patients, regardless of the presence of clinical symptoms.

Finally, data regarding 2D Echo modification reported in 5 -FU trials ${ }^{44,47}$ showed no significant changes in systolic and diastolic function during fluoropyrimidines treatment.

The clinical history of our patient suggests that capecitabine cardiotoxicity may present with different signs and symptoms, even in the same patient, and that the diagnosis may be challenging.

\section{Rechallenge with capecitabine}

Once cardiotoxicity has occurred, the safety of a rechallenge with modified 5-FU- or capecitabine-based regimens is uncertain, and no widely accepted recommendations have been released.

In clinical practice, the reintroduction of a fluoropyrimidinebased chemotherapy with dose reduction together with prophylactic antianginal medications is quite common. However, even if the addition of anti-ischemic drugs, such as calcium channel blockers and nitrates, may reduce the clinical manifestations in some patients, ${ }^{48}$ the chemoprevention of cardiotoxicity with these agents is not definitively proved. For example, in a prospective study including 58 patients who received verapamil during 5-FU therapy, signs of ischemia using electrocardiography appeared as often as in a control group not receiving any antiangina drug. ${ }^{34}$

Since there are not large controlled trials that assessed the efficacy of antiangina therapy in this situation, whether the rechallenge is safe remains controversial ${ }^{49}$ and the option should be discussed on a case-by-case basis.

\section{Conclusion}

In the near future, the incidence of fluoropyrimidineinduced cardiovascular side effects is expected to increase worldwide, because of the large use of these drugs in patients with breast or gastrointestinal cancers, the ageing of the population, and the higher frequency of cardiac comorbid condition in older subjects. The seriousness of this toxicity may vary widely, ranging from asymptomatic electrocardiographic alterations to life-threatening events. Overall, capecitabine cardiac toxicity is reported to affect nearly $5 \%$ of exposed patients, but this figure could underestimate the real incidence due to the underreporting of asymptomatic cases. Even in patients with clinical manifestations, capecitabine-induced cardiac toxicity may be an elusive side effect whose diagnosis is often challenging. Other than preexisting ischemic cardiac disorders, no wellestablished risk factors have been identified. After a first cardiac episode (if mild-to-moderate in severity), fluoropyrimidine rechallenge may be considered, but it should be proposed with great caution. Prophylactic treatment with coronary vasodilators does not seem to prevent such recurrences, although it is often used in clinical practice. If necessary, an active and thoughtful search of any signs or symptoms of cardiac ischemia should be applied. The 
24-hour Holter monitoring on days 4-6 from the start of chemotherapy may be considered an effective and low-cost exam. Patients and caregivers should be educated about this side effect in order to maximize the chance of recognizing early clinical clues of cardiovascular impairment. Moreover, patients should be advised to avoid unusual physical efforts, and to immediately report every cardiac symptom.

\section{Acknowledgment}

Written informed consent was obtained from the patient for publication of this case report and any accompanying images.

\section{Disclosure}

The authors report no conflicts of interest in this work.

\section{References}

1. Malet-Martino M, Jolimaitre P, Martino R. The prodrugs of 5-fluorouracil. Curr Med Chem Anticancer Agents. 2002;2(2):267-310.

2. Aprile G, Mazzer M, Moroso S, Puglisi F. Pharmacology and therapeutic efficacy of capecitabine: focus on breast and colorectal cancer. Anticancer Drugs. 2009;20(4):217-229.

3. Mikhail SE, Sun JF, Marshall JL. Safety of capecitabine: a review. Expert Opin Drug Saf. 2010;9(5):831-841.

4. Albini A, Pennesi G, Donatelli F, Cammarota R, De Flora S, Noonan DM. Cardiotoxicity of anticancer drugs: the need for cardio-oncology and cardio-oncological prevention. J Natl Cancer Inst. 2010;102(1): $14-25$.

5. Polk A, Vaage-Nilsen M, Vistisen K, Nielsen DL. Cardiotoxicity in cancer patients treated with 5 -fluorouracil or capecitabine: a systematic review of incidence, manifestations and predisposing factors. Cancer Treat Rev. 2013;39(8):974-984.

6. Singletary SE, Connolly JL. Breast cancer staging: working with the sixth edition of the AJCC Cancer Staging Manual. CA Cancer J Clin. 2006;56(1):37-47; quiz 50-51.

7. Zambetti M, Mansutti M, Gomez P, et al. Pathological complete response rates following different neoadjuvant chemotherapy regimens for operable breast cancer according to ER status, in two parallel, randomized phase II trials with an adaptive study design (ECTO II). Breast Cancer Res Treat. 2012;132(3):843-851.

8. Geyer CE, Forster J, Lindquist D, et al. Lapatinib plus capecitabine for HER2-positive advanced breast cancer. $N$ Engl J Med. 2006;355(26): 2733-2743.

9. Imazio M, Trinchero R. Myopericarditis: Etiology, management, and prognosis. Int J Cardiol. 2008;127(1):17-26.

10. Gianni M, Dentali F, Lonn E. 5 flourouracil-induced apical ballooning syndrome: a case report. Blood Coagul Fibrinolysis. 2009;20(4): 306-308.

11. Kobayashi N, Hata N, Yokoyama S, Shinada T, Shirakabe A, Mizuno K. A case of Takotsubo cardiomyopathy during 5-fluorouracil treatment for rectal adenocarcinoma. J Nippon Med Sch. 2009;76(1):27-33.

12. Stewart T, Pavlakis N, Ward M. Cardiotoxicity with 5-fluorouracil and capecitabine: more than just vasospastic angina. Intern Med J. 2010;40(4):303-307.

13. Y-Hassan S, Tornvall P, Törnerud M, Henareh L. Capecitabine caused cardiogenic shock through induction of global Takotsubo syndrome. Cardiovasc Revasc Med. 2013;14(1):57-61.

14. Kurowski V, Kaiser A, von Hof K, et al. Apical and midventricular transient left ventricular dysfunction syndrome (tako-tsubo cardiomyopathy): frequency, mechanisms, and prognosis. Chest. 2007;132(3):809-816.
15. Simmons A, Vacek JL, Meyers D. Anthracycline-induced cardiomyopathy. Postgrad Med. 2008;120(4):67-72.

16. Ferrans VJ, Clark JR, Zhang J, Yu ZX, Herman EH. Pathogenesis and prevention of doxorubicin cardiomyopathy. Tsitologiia. 1997;39(10): 928-937.

17. Camaro C, Danse PW, Bosker HA. Acute chest pain in a patient treated with capecitabine. Neth Heart J. 2009;17(7-8):288-291.

18. Kusama Y, Kodani E, Nakagomi A, et al. Variant angina and coronary artery spasm: the clinical spectrum, pathophysiology, and management. J Nippon Med Sch. 2011;78(1):4-12.

19. Südhoff T, Enderle MD, Pahlke M, et al. 5-Fluorouracil induces arterial vasocontractions. Ann Oncol. 2004;15(4):661-664.

20. Goldsmith YB, Roistacher N, Baum MS. Capecitabine-induced coronary vasospasm. J Clin Oncol. 2008;26(22):3802-3804.

21. Golias C, Dimitriadis G, Dimitriadis D, et al. Acute presentation of vasospastic angina induced by oral capecitabine: a case report. $J$ Med Case Rep. 2014;8:18.

22. Kuppens IE, Boot H, Beijnen JH, Schellens JH, Labadie J. Capecitabine induces severe angina-like chest pain. Ann Intern Med. 2004;140(6): 494-495.

23. Shah NR, Shah A, Rather A. Ventricular fibrillation as a likely consequence of capecitabine-induced coronary vasospasm. J Oncol Pharm Pract. 2012;18(1):132-135.

24. Fradley MG, Barrett CD, Clark JR, Francis SA. Ventricular fibrillation cardiac arrest due to 5-fluorouracil cardiotoxicity. Tex Heart Inst J. 2013;40(4):472-476.

25. Wang K, Asinger RW, Marriott HJ. ST-segment elevation in conditions other than acute myocardial infarction. N Engl J Med. 2003;349(22): $2128-2135$.

26. Lestuzzi C, Tartuferi L, Corona G. Capecitabine (and 5 fluorouracil) cardiotoxicity. Metabolic considerations. Breast J. 2011;17(5):564-565; author reply $566-567$.

27. Lamberti M, Porto S, Zappavigna S, et al. A mechanistic study on the cardiotoxicity of 5-fluorouracil in vitro and clinical and occupational perspectives. Toxicol Lett. 2014;227(3):151-156.

28. Van Cutsem E, Hoff PM, Blum JL, Abt M, Osterwalder B. Incidence of cardiotoxicity with the oral fluoropyrimidine capecitabine is typical of that reported with 5-fluorouracil. Ann Oncol. 2002;13(3): 484-485.

29. Kosmas C, Kallistratos MS, Kopterides P, et al. Cardiotoxicity of fluoropyrimidines in different schedules of administration: a prospective study. J Cancer Res Clin Oncol. 2008;134(1):75-82.

30. Lestuzzi C, Viel E, Picano E, Meneguzzo N. Coronary vasospasm as a cause of effort-related myocardial ischemia during low-dose chronic continuous infusion of 5-fluorouracil. Am J Med. 2001;111(4): 316-318.

31. Miura K, Kinouchi M, Ishida K, et al. 5-fu metabolism in cancer and orally-administrable 5-fu drugs. Cancers (Basel). 2010;2(3): $1717-1730$.

32. Mosseri M, Fingert HJ, Varticovski L, Chokshi S, Isner JM. In vitro evidence that myocardial ischemia resulting from 5 -fluorouracil chemotherapy is due to protein kinase C-mediated vasoconstriction of vascular smooth muscle. Cancer Res. 1993;53(13): 3028-3033.

33. Shoemaker LK, Arora U, Rocha Lima CM. 5-fluorouracil-induced coronary vasospasm. Cancer Control. 2004;11(1):46-49.

34. Eskilsson J, Albertsson M. Failure of preventing 5-fluorouracil cardiotoxicity by prophylactic treatment with verapamil. Acta Oncol. 1990;29(8):1001-1003.

35. Patel B, Kloner RA, Ensley J, Al-Sarraf M, Kish J, Wynne J. 5-Fluorouracil cardiotoxicity: left ventricular dysfunction and effect of coronary vasodilators. Am J Med Sci. 1987;294(4):238-243.

36. Kinhult S, Albertsson M, Eskilsson J, Cwikiel M. Antithrombotic treatment in protection against thrombogenic effects of 5-fluorouracil on vascular endothelium: a scanning microscopy evaluation. Scanning. 2001;23(1):1-8. 
37. De Forni M, Malet-Martino MC, Jaillais P, et al. Cardiotoxicity of high-dose continuous infusion fluorouracil: a prospective clinical study. $J$ Clin Oncol. 1992;10(11):1795-1801.

38. Jensen SA, Sørensen JB. Risk factors and prevention of cardiotoxicity induced by 5-fluorouracil or capecitabine. Cancer Chemother Pharmacol. 2006;58(4):487-493.

39. Meyer CC, Calis KA, Burke LB, Walawander CA, Grasela TH. Symptomatic cardiotoxicity associated with 5-fluorouracil. Pharmacotherapy. 1997;17(4):729-736.

40. Koca D, Salman T, Unek IT, et al. Clinical and electrocardiography changes in patients treated with capecitabine. Chemotherapy. 2011;57(5):381-387.

41. Ignatescu MC, Gharehbaghi-Schnell E, Hassan A, et al. Expression of the angiogenic protein, platelet-derived endothelial cell growth factor in coronary atherosclerotic plaques: In vivo correlation of lesional microvessel density and constrictive vascular remodeling. Arterioscler Thromb Vasc Biol. 1999;19(10):2340-2347.

42. Lestuzzi C, Vaccher E, Talamini R, et al. Effort myocardial ischemia during chemotherapy with 5-fluorouracil: an underestimated risk. Ann Oncol. 2014;25(5):1059-1064.

43. Ng M, Cunningham D, Norman AR. The frequency and pattern of cardiotoxicity observed with capecitabine used in conjunction with oxaliplatin in patients treated for advanced colorectal cancer (CRC) Eur J Cancer. 2005;41(11):1542-1546.
44. Oztop I, Gencer M, Okan T, et al. Evaluation of cardiotoxicity of a combined bolus plus infusional 5-fluorouracil/folinic acid treatment by echocardiography, plasma troponin I level, QT interval and dispersion in patients with gastrointestinal system cancers. Jpn J Clin Oncol. 2004;34(5):262-268.

45. Salepci T, Seker M, Uyarel H, et al. 5-Fluorouracil induces arterial vasoconstrictions but does not increase angiotensin II levels. Med Oncol. 2010;27(2):416-420.

46. Holubec L Jr, Topolcan O, Finek J, et al. Dynamic monitoring of cardio-specific markers and markers of thyroid gland function in cancer patients - a pilot study. Anticancer Res. 2007;27(4A):1883-1886.

47. Ceyhan C, Meydan N, Barutca S, et al. Ultrasound tissue characterization by integrated backscatter for analyzing Fluorouracil induced myocardial damage. Echocardiography. 2005;22(3):233-238.

48. Cianci G, Morelli MF, Cannita K, et al. Prophylactic options in patients with 5-fluorouracil-associated cardiotoxicity. $\mathrm{Br} J$ Cancer. 2003;88(10):1507-1509.

49. Labianca R, Luporini G. 5-fluorouracil cardiotoxicity: the risk of rechallenge. Ann Oncol. 1991;2(5):383.

\section{Publish your work in this journal}

OncoTargets and Therapy is an international, peer-reviewed, open access journal focusing on the pathological basis of all cancers, potential targets for therapy and treatment protocols employed to improve the management of cancer patients. The journal also focuses on the impact of management programs and new therapeutic agents and protocols on

\section{Dovepress}

patient perspectives such as quality of life, adherence and satisfaction. The manuscript management system is completely online and includes a very quick and fair peer-review system, which is all easy to use. Visit http://www.dovepress.com/testimonials.php to read real quotes from published authors. 